\title{
Une expérience simple pour comprendre les rétroactions climatiques positives
}

\author{
Venance Journé, Jean-Louis Dufresne \\ Laboratoire de météorologie dynamique, Institut Pierre-Simon Laplace, CNRS / \\ Sorbonne Université, Paris \\ venance.journe@Imd.jussieu.fr
}

But pédagogique : expliquer le fonctionnement d'une rétroaction par une expérience simple, montrer qu'une rétroaction positive ne conduit pas forcément à un emballement et faire le lien avec des rétroactions dans le système climatique.

Public: lycée, années préparatoires aux grandes écoles et premières années d'université.

Matériel nécessaire: une potence, des baguettes de bois, deux cylindres en plastique transparent comportant deux ouvertures, un tuyau flexible, un robinet d'arrêt, une tige de métal, un disque en plexiglas, une petite bouteille en plastique. Une vidéo présentant le déroulement de l'expérience est disponible sur le site de la revue.

\section{Principe de la rétroaction}

Dans la nature, les systèmes sont composés de différents éléments qui interagissent entre eux. Une action sur un élément du système peut produire une réaction sur un autre élément du système qui peut à son tour réagir. Si les deux éléments sont liés, ou "couplés», de façon à ce que la réaction du second élément affecte le premier, on parle de rétroaction. Une rétroaction suppose que les deux éléments agissent l'un sur l'autre en réponse à une perturbation du système; la réponse du second élément agit (action) en retour (rétro) sur la réponse du premier élément. Il est important de noter que la rétroaction fait partie intégrante du système, qu'elle est toujours à l'œuvre. Isoler l'effet d'une rétroaction nécessite de modifier le système à l'étude soit directement comme dans l'expérience présentée, soit, ce qui est le plus fréquent, par des calculs.

On utilisera le terme «perturbation» pour caractériser une action qui modifie l'état d'équilibre d'un système. Une rétroaction est positive si elle augmente l'effet de la perturbation et négative si elle le diminue.

La cybernétique, qui est la science du contrôle des systèmes, a été inventée en 1948 par le mathématicien américain Norbert Wiener qui s'était intéressé aux armes anti-aériennes et aux prothèses médicales. Il est le premier à avoir formalisé le concept de «rétroaction» (ou feedback en anglais). D'après de Rosnay (1978), Norbert Wiener travaillait en 1940 avec un jeune ingénieur, Julian H. Bigelow, sur la mise au point d'appareils de pointage automatique pour des canons antiaériens. Les servomécanismes sont capables d'anticiper la trajectoire d'un avion en prenant en compte la trajectoire passée. Norbert Wiener, étonné et impressionné par le fonctionnement apparemment intelligent de ces machines, en déduisit que, pour contrôler une action finalisée, la circulation de l'information nécessaire à ce contrôle doit former «une boucle fermée permettant l'évaluation des effets de ses actions et l'adaptation de la conduite future à partir des performances passées $\gg$. Wiener et Bigelow avaient découvert la boucle d'information nécessaire pour corriger une action, la boucle de rétroaction.

Les exemples de rétroaction sont nombreux dans les systèmes physiques, biologiques et sociaux. Un exemple simple en physique est celui d'une casserole d'eau qui bout: l'eau n'atteint jamais la température de la flamme, car l'évaporation lui fait perdre de la chaleur et maintient la température au voisinage de $100{ }^{\circ} \mathrm{C}$. 
$\mathrm{Si}$ on augmente la puissance de chauffage, ce qui correspond à introduire une perturbation, l'eau s'évaporera plus rapidement. Cependant, la température restera quasiment constante, l'effet de la perturbation sur la température est donc quasi nul. C'est un exemple de rétroaction négative, qui durera autant de temps qu'il y aura de l'eau dans la casserole. Dans le domaine biologique, les rétroactions permettent au corps humain ou aux écosystèmes de garder leur équilibre en maintenant les paramètres vitaux dans une gamme de valeurs qui leur permet de rester en bonne santé, comme la pression artérielle ou celle de la température, qui est notamment régulée par la transpiration. Comme l'écrit François Jacob (1970): «Toute organisation fait intervenir des boucles de régulation par quoi chaque élément est tenu informé des effets de son propre fonctionnement et l'ajuste en conséquence dans l'intérêt du tout.»

\section{La rétroaction de l'albédo de surface}

Préalablement à la description et à l'explication de l'expérience, nous présentons une rétroaction du système climatique dont le mécanisme peut être exposé simplement.

Le système Terre (composé de l'océan, de l'atmosphère et des surfaces continentales) reçoit toute son énergie sous la forme de rayonnement du Soleil et perd son énergie par émission de rayonnement infrarouge. En dehors de toute perturbation, et sur des durées assez longues, de l'ordre de centaines ou de milliers d'années, le bilan radiatif du système Terre s'équilibre au sommet de l'atmosphère.

Une partie de l'énergie solaire incidente est réfléchie par les gaz de l'atmosphère, les aérosols (poussières, etc.), les nuages et les différents éléments à la surface de la Terre. On appelle albédo planétaire le rapport entre l'énergie solaire réfléchie et l'énergie solaire incidente au sommet de l'atmosphère et à l'échelle de la planète (voir encadré 1).

Supposons tout d'abord que l'albédo soit constant. Une augmentation du flux solaire incident entraînerait une augmentation de l'énergie solaire absorbée, de la température et donc de l'énergie perdue par émission de rayonnement infrarouge jusqu'à ce qu'un nouvel équilibre soit atteint et que l'énergie perdue soit égale à l'énergie absorbée.

Supposons maintenant que l'albédo varie avec la température et explicitons la rétroaction entre les variations de température et d'albédo.

Un exemple de rétroaction positive est celui de la fonte de la glace de mer: si la température s'élève, la glace fond, réduisant ainsi l'extension de la glace de mer (très réfléchissante) et augmentant donc la surface de l'eau libre (très absorbante). L'énergie réfléchie par la glace de mer diminue, celle absorbée par l'océan augmente : la Terre reçoit en moyenne davantage d'énergie. L'absorption d'énergie solaire entraîne également une augmentation de la température, qui favorise la diminution de la glace de mer, et ainsi de suite. C'est l'exemple typique d'une rétroaction positive dont le mécanisme pourrait se prolonger jusqu'à ce que la glace de mer au pôle Nord ait complètement disparu.

Imaginons maintenant un exemple pour lequel l'albédo augmenterait avec la température. Cela pourrait être le cas d'une végétation sombre au-dessus d'un sol plus clair. Si la température s'élève et que le couvert végétal, en situation de sécheresse, diminue, la fraction de sol

\section{Les valeurs de l'albédo}

La valeur locale de l'albédo est très variable suivant la nature de la surface terrestre. Approximativement, elle est de $90 \%$ pour la neige, $60 \%$ pour la glace de mer, $30 \%$ pour un sol sableux, 5 à $20 \%$ pour les forêts et $10 \%$ pour l'océan. À l'échelle du globe, on parle d'albédo planétaire. Celui-ci est influencé par de nombreux facteurs. Suivant les saisons, les surfaces continentales ou océaniques recouvertes de neige et de glace, ayant un fort pouvoir réfléchissant, diminuent ou augmentent. Les nuages ont un pouvoir réfléchissant et la couverture nuageuse varie également en fonction des saisons. Des éruptions volcaniques peuvent libérer de grandes quantités d'aérosols dans l'atmosphère qui produisent un effet réfléchissant pendant plusieurs années, ce qui affectera aussi l'albédo. La valeur de l'albédo du système Terre est déterminée à partir de nombreuses observations satellitaires depuis les années 1970. Elle est estimée à environ 0,3 , c'est-à-dire que $30 \%$ environ de l'énergie reçue du Soleil est réfléchie par l'atmosphère et la surface de la Terre. nu plus clair augmente, ce qui augmente l'albédo. Le flux solaire est davantage réfléchi, ce qui réduit l'augmentation de température par rapport à une situation où l'albédo serait constant. C'est un exemple typique de rétroaction négative.

Pour notre planète, cette rétroaction négative de l'albédo est minoritaire par rapport à la rétroaction positive liée à la glace de mer et, de façon plus générale, à la cryosphère dans son ensemble.

L'encadré 2 explicite la variation de la température en réponse à une variation du flux solaire avec et sans rétroaction, faisant apparaître la différence de comportement selon que l'albédo augmente ou diminue lorsque la température augmente.

\section{Description de l'expérience}

Le but de l'expérience proposée est de visualiser de façon simple un couplage et une rétroaction dans un système perturbé, de montrer qu'un système peut être stable même en présence d'une rétroaction positive et que celle-ci n'entraîne pas obligatoirement un emballement (Dufresne et Saint-Lu, 2016). Le principe de l'expérience est de mettre en évidence le couplage entre deux processus simples: l'équilibre d'une balance d'une part, l'écoulement d'un fluide entre les deux bras de la balance d'autre part.

La figure 4 montre une schématisation du dispositif, qui est simplifié pour en faciliter la description mathématique. En particulier, les récipients cylindriques sont verticaux sur le dessin alors qu'ils sont solidaires des bras dans le dispositif que nous avons construit. Ce dispositif consiste en un balancier composé d'un bras horizontal fixé en son milieu $\mathrm{O}$ à un bras vertical, le tout formant un T. Le balancier est accroché à une barre verticale fixe en son point $\mathrm{O}$ autour duquel il peut pivoter. Deux récipients cylindriques contenant de l'eau, de masse $m_{2}$, sont fixés sur le bras horizontal, séparés d'une distance $l_{2}$, chacun à une distance identique de part et d'autre du point $\mathrm{O}$. Les deux récipients sont reliés en haut et en bas par des tuyaux. Le système constitué des cylindres et des tuyaux est étanche. Le tuyau inférieur est muni d'un robinet qui permet d'interrompre la circulation d'eau entre les deux cylindres. 


\section{Calcul de la variation de la température avec le flux solaire, sans et avec rétroaction de l'albédo de surface}

Considérons l'effet d'une variation du flux solaire sur la température. La puissance reçue du Soleil s'écrit:

$$
I_{0} \pi R^{2}
$$

où $I_{0}$ est la constante solaire, c'est-à-dire la puissance reçue du Soleil par mètre carré du système Terre $\left(1360 \mathrm{~W} / \mathrm{m}^{2}\right)$ et $R$ est le rayon de la Terre.

Si l'on considère que le système Terre se comporte dans le domaine infrarouge comme un corps noir parfait sphérique, la puissance qu'il émet s'écrit:

$$
4 \pi R^{2} \sigma T^{4}
$$

où $\sigma T^{4}$ est la puissance émise par le système Terre d'après la loi de Stefan-Boltzmann, $T$ étant la température du système Terre en kelvins et $\sigma=5,6710^{-8} \mathrm{~W} \mathrm{~m}^{-2} \mathrm{~K}^{-4}$.

En simplifiant, on peut écrire que, en régime stationnaire, le flux solaire absorbé par unité de surface $F_{a}$ est égal à celui émis par le système Terre et s'écrit:

$$
F_{\mathrm{a}}=\sigma T^{4}
$$

En conséquence, pour de faibles variations, une variation du flux solaire absorbé $\Delta F_{\mathrm{a}}$ entraîne une variation de température $\Delta T$ :

$$
\Delta T=\frac{\Delta F_{\mathrm{a}}}{4 \sigma T^{3}}
$$

En définissant un paramètre de réponse $\lambda$ tel que $\Delta T=\frac{\Delta F}{-\lambda}$, l'équation se réécrit sous la forme:

$$
\Delta T=\frac{\Delta F_{\mathrm{a}}}{-\lambda_{\mathrm{p}}}
$$

où $\lambda_{\mathrm{p}}$ est ce que l'on appelle le paramètre de réponse de Planck, qui s'écrit:

$$
\lambda_{\mathrm{p}}=-4 \sigma T^{3}=\frac{\Delta F_{\mathrm{e}}}{\Delta T}
$$

où $\Delta F_{\mathrm{e}}$ est la variation de flux émis.

Faisons maintenant le lien avec l'albédo. Le flux solaire absorbé est:

$$
F_{\mathrm{a}}=\sigma T^{4}=\frac{I_{0}(1-A)}{4}
$$

où $A$ est l'albédo planétaire.

Pour une simplification des formules, on utilisera

$$
\begin{gathered}
I=\frac{I_{0}}{4} \\
F_{\mathrm{a}}=I(1-A)
\end{gathered}
$$

S'il n'y a pas de couplage entre l'albédo et la température ou si l'albédo ne varie pas avec la température, une perturbation sur / se traduit par une perturbation sur le flux solaire absorbé, qui s'écrit:

$$
\Delta F_{\mathrm{ai}}=(1-A) \Delta l
$$

Dans ce cas, le changement de température suite à la réponse de Planck est:

$$
\Delta T_{p}=(1-A) \frac{\Delta l}{-\lambda_{p}}
$$

Considérons le cas où l'albédo dépend de la température, alors une modification de celle-ci peut entraîner une variation de l'albédo. La variation du flux solaire absorbé $\Delta F_{\mathrm{a}}$ peut alors être décomposée en deux termes: $\Delta F_{\mathrm{ai}}$ la variation du flux solaire absorbé en l'absence de rétroaction, et $\Delta F_{\text {ra }}$, un terme supplémentaire dû à la rétroaction de l'albédo de surface:

$$
\Delta F_{\mathrm{a}}=\Delta F_{\mathrm{ai}}+\Delta F_{\mathrm{ra}}
$$

La variation du flux solaire absorbé $\Delta F_{\text {ra }}$ dépendant de la variation de l'albédo en fonction de la température $\frac{\delta A}{\delta T}$, on peut écrire:

$$
\Delta F_{\mathrm{ra}}=-1 \frac{\delta A}{\delta T} \Delta T
$$

Donc, la variation totale du flux solaire absorbé s'écrit:

$$
\Delta F_{\mathrm{a}}=(1-A) \Delta I-I \frac{\delta A}{\delta T} \Delta T
$$

et, rappelant que $\Delta T=\frac{\Delta F_{\mathrm{a}}}{-\lambda_{\mathrm{p}}}$, la variation de
la température s'écrit:

$$
\Delta T=\left[(1-A) \frac{\Delta I}{-\lambda_{p}}\right]-\left[I \frac{\delta A}{\delta T} \frac{\Delta T}{-\lambda_{p}}\right]
$$

En écrivant $\lambda_{\mathrm{a}}$ le paramètre de réponse albédo:

$$
\lambda_{\mathrm{a}}=-\mathrm{I} \frac{\delta A}{\delta T}=\frac{\Delta F_{\mathrm{ra}}}{\Delta T}
$$

L'équation se réécrit:

$$
\begin{gathered}
\Delta T=\Delta T_{\mathrm{p}}-\Delta T \frac{\lambda_{\mathrm{a}}}{\lambda_{\mathrm{p}}} \\
\text { soit } \Delta T=\frac{1}{1+\frac{\lambda_{\mathrm{a}}}{\lambda_{\mathrm{p}}}} \Delta T_{\mathrm{p}}
\end{gathered}
$$

La variation de température $\Delta T$ avec rétroaction est égale à celle de $\Delta T_{\mathrm{p}}$ sans rétroaction multipliée par $\frac{1}{1+\frac{\lambda_{a}}{\lambda_{p}}}$

Le paramètre $\lambda_{\mathrm{p}}$ étant négatif, une valeur positive de $\lambda_{a}$ entraînera un effet amplificateur de la rétroaction

$\lambda_{a}>0 \Rightarrow \frac{\lambda_{a}}{\lambda_{p}}<0 \Rightarrow 1+\frac{\lambda_{a}}{\lambda_{p}}<1 \Rightarrow \frac{1}{1+\frac{\lambda_{a}}{\lambda_{p}}}>1$

et une valeur négative, un effet atténuateur. En d'autres termes, si l'albédo diminue lorsque la température augmente (cas de la neige qui fond lorsque la température augmente),

$$
\frac{\delta A}{\delta T}<0,
$$

donc $\lambda_{a}>0$ : il s'agit d'une situation où la rétroaction amplifie l'effet de la perturbation. Si l'albédo augmente avec la température $\left(\frac{\delta A}{\delta T}>0\right)$, c'est la situation contraire.

Ce même raisonnement pourrait s'appliquer à un refroidissement.
De façon à pouvoir perturber l'équilibre du système, il est possible d'accrocher une masse $m_{1}$ à l'une des extrémités du bras horizontal, à la distance $l_{1}$ du point $\mathrm{O}$.

Une masse $m_{3}$ est accrochée en bas du bras vertical à une distance $l_{3}$ du point $\mathrm{O}$ et sert de contrepoids pour limiter la déviation du balancier due à la perturbation.

$\mathrm{Au}$ départ, les cylindres contiennent la même quantité d'eau et sont remplis à la moitié environ. Le robinet est fermé: les cylindres ne peuvent communiquer. Aucune masse n'est accrochée au bras portant les cylindres d'eau, il reste horizontal. Le balancier est à l'équilibre (figure 5a).

L'expérience se déroule en trois phases.

Première phase. Une masse $m_{1}$ est accrochée à une extrémité du bras horizontal sur lequel sont fixés les cylindres, ce qui modifie l'équilibre. Le système réagit à la perturbation et le bras de la balance est dévié par rapport à la position de départ horizontale. La masse $m_{3}$ accrochée au bras vertical exerce une force de rappel qui permet de stabiliser le système. Le bras vertical fait initialement un angle $\Delta \theta$ avec la barre fixe verticale. Tant que les deux cylindres ne peuvent « communiquer »- il n'y a pas de couplage entre la déviation des bras de la balance et l'écoulement d'eau entre les deux cylindres -, le système se stabilise rapidement à un nouvel état: le moment résultant des deux poids des masses $m_{1}$ et $m_{3}$ s'annule. Si la masse $m_{1}$ qui perturbe l'équilibre est relativement faible par rapport à la masse $m_{3}$ qui sert de contrepoids, l'angle de déviation est faible (figure 5b). Les figures $5 \mathrm{c}$ et $5 \mathrm{~d}$ montrent que l'angle de déviation augmente avec la masse de la perturbation, ici doublée puis triplée.

Deuxième phase. On recommence en ouvrant le robinet. La masse $m_{1}$ est mise en place, l'eau se met à circuler, il y a couplage entre les deux processus : l'inclinaison du balancier et la circulation de l'eau. Lors de la démonstration, on peut demander à ce moment-là aux spectateurs leur avis sur ce qu'il va se produire: la déviation angulaire va-t-elle rester constante, augmenter ou diminuer? Par gravité, l'eau s'écoule du cylindre du haut vers celui qui est en bas: le cylindre du bas se remplit, le système penche davantage, l'angle $\theta$ augmente, la distance verticale entre les deux cylindres augmente, ce qui entraîne 


\section{Montage de l'expérience}

Le support au sol est une plaque de bois de $25 \times 15 \mathrm{~cm}^{2}$. Sur cette plaque est fixé un montant en bois de $1,20 \mathrm{~m}$ de hauteur de section carrée de $4 \mathrm{~cm}$ de côté. Une tige métallique est fixée à une hauteur de $1,05 \mathrm{~m}$ et dépasse de $8 \mathrm{~cm}$ (figure 1). Cette tige de métal sera insérée dans un trou du balancier et lui servira d'axe de rotation 0 (figure 2).

Le balancier en forme de $T$ est constitué de trois pièces de bois (figure 5 ):

- deux baguettes de $50 \mathrm{~cm}$ de longueur de section carrée de $1 \mathrm{~cm}$ de côté fixées l'une sur l'autre de façon à faire un $\mathrm{T}$ bien symétrique;

- une plaque de bois de $5 \times 9 \mathrm{~cm}^{2}$ et de $1 \mathrm{~cm}$ d'épaisseur, fixée en haut du bras vertical et dans laquelle est percée le trou qui recevra la tige métallique (figure 2).

Une petite plaque en plexiglas transparent (un rapporteur) permettant de mesurer la déviation angulaire du balancier est fixée au milieu de la tige horizontale du balancier (figure 2).

Des plaques de métal seront attachées à une extrémité du bras horizontal du balancier. Ces poids feront pencher le balancier en perturbant son équilibre.

Une petite bouteille en plastique d'un demilitre est accrochée en bas du bras vertical du balancier. Cette bouteille, partiellement remplie d'eau (au tiers environ), agira comme contrepoids pour limiter la déviation angulaire du balancier (voir figure 5). L'intérêt d'utiliser une bouteille d'eau est de pouvoir faire facilement varier la valeur du contrepoids.

Deux contenants cylindriques en plastique de $24 \mathrm{~cm}$ de hauteur et de $6 \mathrm{~cm}$ de diamètre, strictement identiques, sont fixés de façon symétrique sur le bras horizontal du balancier. Ces contenants sont partiellement remplis d'eau (figure 3 ), ici environ à la moitié.

Ces deux contenants sont hermétiquement reliés en haut et en bas par deux tuyaux flexibles : le tuyau du bas étant muni d'un robinet d'arrêt pour isoler les deux cylindres (figure 3).

Nous indiquons les mesures du dispositif tel que nous l'avons construit. Elles peuvent être modifiées et l'expérimentateur pourra alors observer les changements des conditions de la rétroaction, comme par exemple l'influence, sur la déviation angulaire du balancier, de la longueur du bras horizontal et du contrepoids ou la quantité d'eau dans les deux récipients cylindriques. Tous les matériaux nécessaires à sa fabrication (bois, tuyaux flexibles et robinet d'arrêt) peuvent être facilement trouvés dans des magasins de bricolage ou d'animalerie: les deux récipients cylindriques sont des réservoirs d'eau pour hamsters.

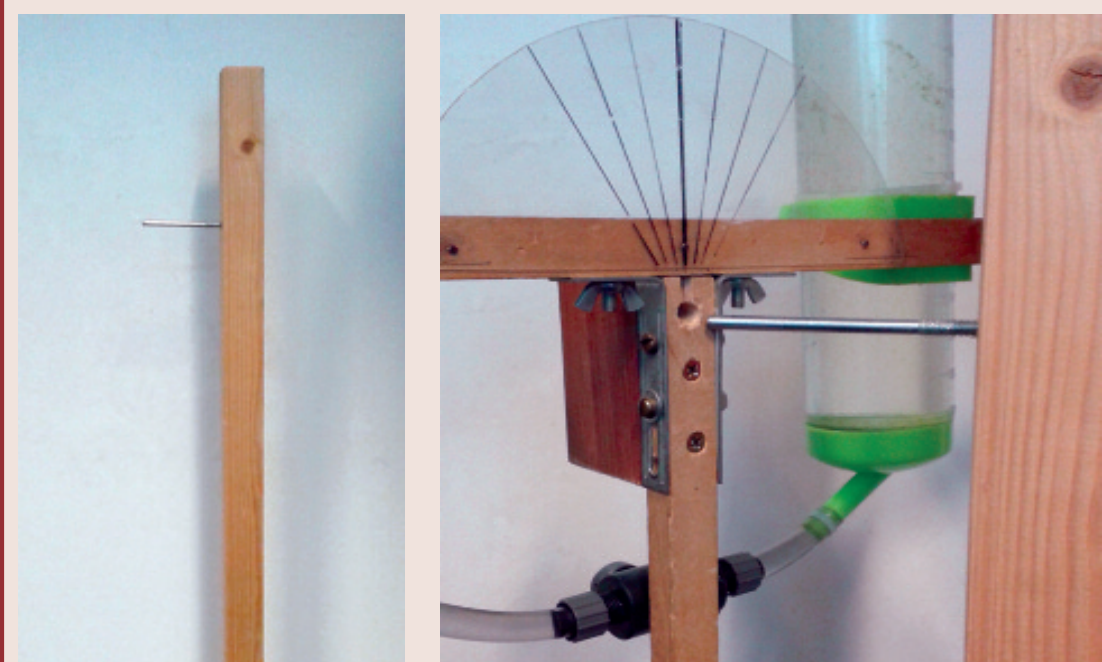

Figure 2. Tige de métal à insérer dans un trou du balancier.

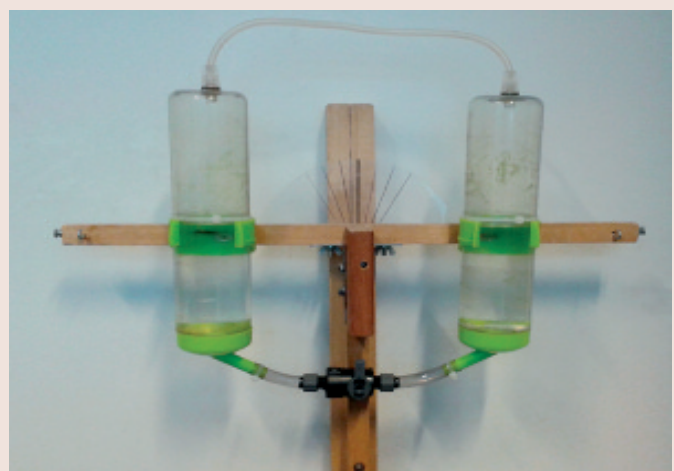

Figure 3. Détail du balancier avec les deux cylindres reliés par des tuyaux flexibles dont un est muni d'un robinet.

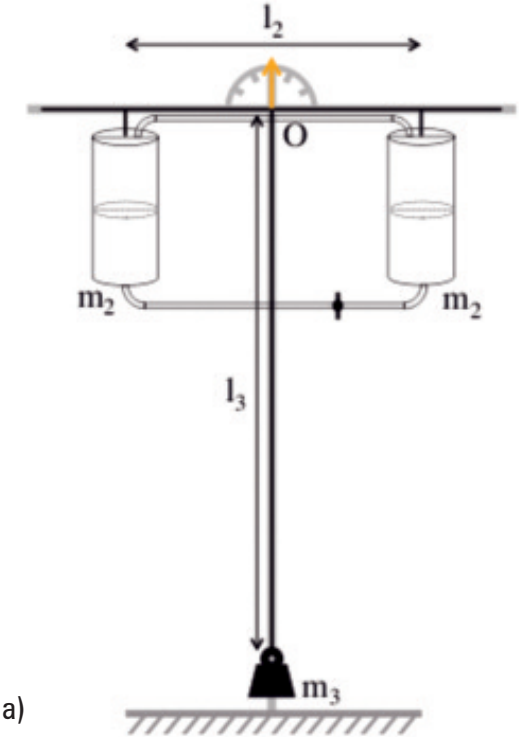

b)
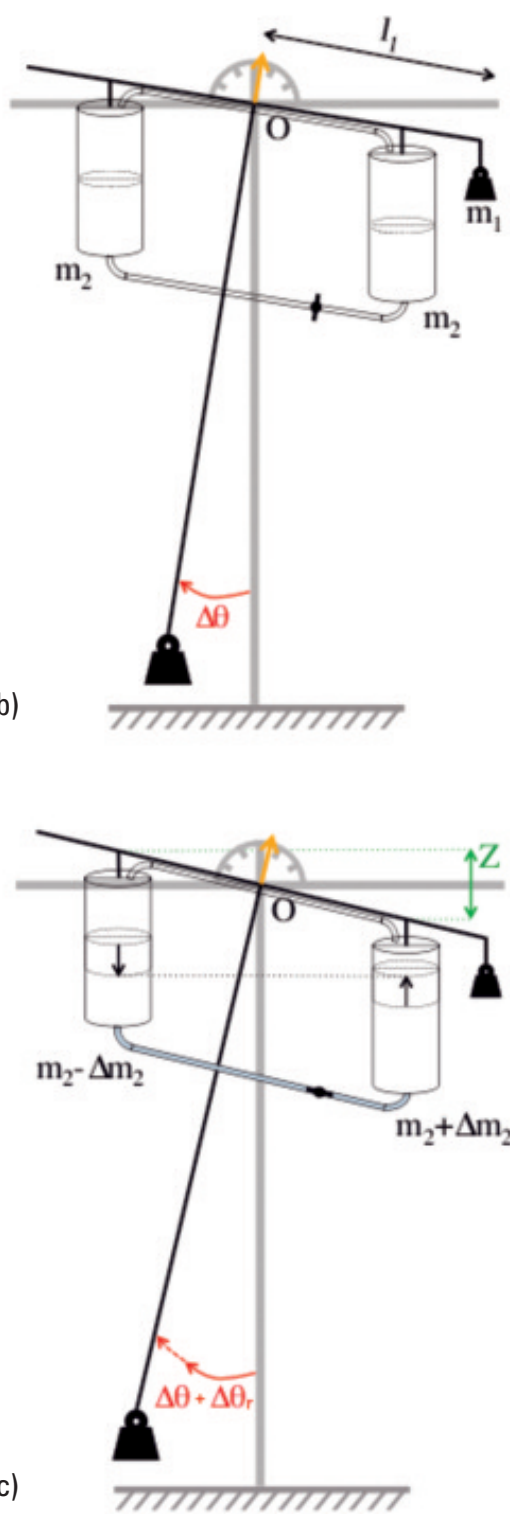

Figure 4. Schéma du dispositif expérimental (d'après Dufresne et Saint-Lu, 2016) : a) système non perturbé ; b) système perturbé, robinet fermé, les deux cylindres d'eau ne communiquent pas ; c) système perturbé, robinet ouvert, les deux cylindres d'eau communiquent. 


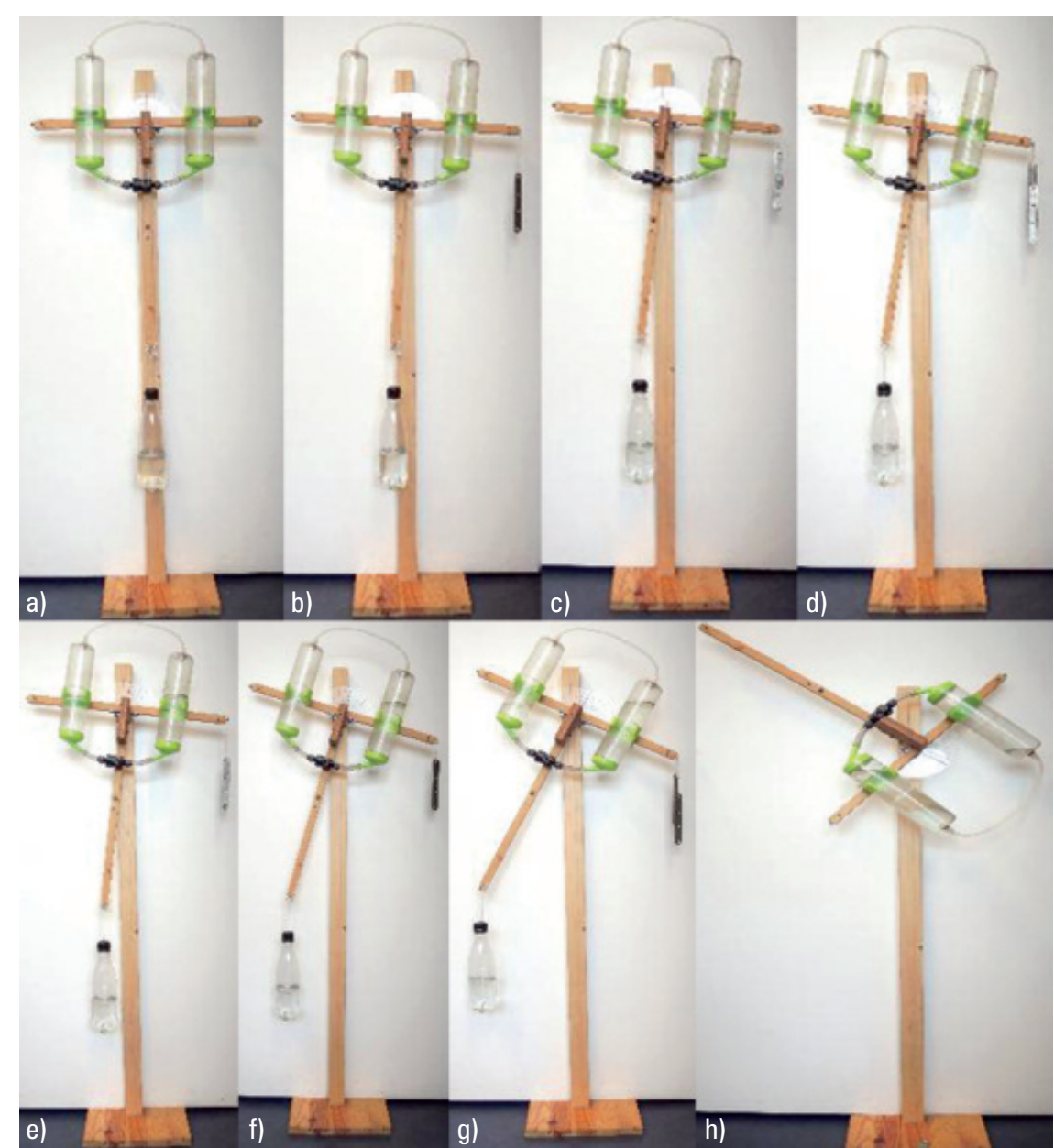

Figure 5. Dispositif expérimental : a) état initial, système non perturbé ; b) avec perturbation (une masse $m_{1}=M$ est accrochée à une extrémité du bras horizontal), le robinet est fermé, les deux cylindres ne sont pas liés ; c) idem avec la masse $m_{1}=2 \mathrm{M}$; d) idem avec la masse $m_{1}=3 \mathrm{M}$; e) avec perturbation (masse $m_{1}=M$ ), le robinet est ouvert, les deux cylindres communiquent, le cylindre du haut se vide, celui du bas se remplit, nouvel état stable ; f) idem avec la masse $m_{1}=$ $2 M ; g)$ idem avec la masse $m_{1}=3 M ; h$ ) avec perturbation, le contrepoids a été enlevé

davantage d'eau dans le cylindre du bas et ainsi de suite: il y a amplification de l'effet initial, c'est une rétroaction positive (figure 5e). En doublant ou triplant la masse de la perturbation, l'amplitude de la déviation augmente (figures $5 \mathrm{f}$ et $5 \mathrm{~g}$ ). Il est conseillé d'attendre suffisamment de temps afin que le nouvel équilibre puisse s'établir. On voit bien que la rétroaction conduit à une modification importante de la déviation angulaire, que le système se trouve dans un état très différent de l'état initial, mais que le nouvel état est stable.

En faisant varier les conditions expérimentales, par exemple en faisant varier la masse d'eau dans les cylindres, la masse de la perturbation ou encore celle du contrepoids, on remarque que le système peut s'immobiliser dans les trois cas suivants :

- la masse de rappel $m_{3}$ ayant un effet stabilisateur sur le système, plus celuici penche, plus la force de rappel due à cette masse est importante. Suivant le réglage (valeur de $m_{3}$ ou de $l_{3}$ ), il est possible que le système s'immobilise avant que le cylindre du haut ne soit vidé (figure 5) ;

- le cylindre bas se remplit graduellement jusqu'à ce que celui du haut soit vide: l'eau ne peut plus couler et le système s'immobilise. C'est le cas de la figure 6. Par rapport au dispositif de la figure 5, la seule différence est que le contrepoids a été diminué d'environ un quart ;

- il y a suffisamment d'eau pour remplir plus d'un cylindre ; alors celui du bas se remplit complètement et il n'y a plus de place pour un écoulement supplémentaire. La rétroaction est saturée.

Troisième phase. Ce qui précède est valable si la masse de rappel $m_{3}$ est suffisante. Si ce n'est pas le cas (s'il n'y a pas assez d'eau dans la bouteille) ou si la masse de rappel $m_{3}$ est enlevée (cas extrême, mais très illustratif), le balancier se retourne (figure $5 \mathrm{~h}$ ). L'augmentation de moment dû au transfert de l'eau entre les réservoirs n'est pas compensée par celui de la masse $m_{3}$ qui sert de contrepoids. Le système est instable, une toute petite perturbation entraîne le système dans un état très différent de l'initial. Cela montre que cette masse a un effet stabilisateur: un état d'équilibre avec une rétroaction positive n'est possible que si un autre phénomène compense la rétroaction.

Une description mathématique de cette expérience est présentée dans l'encadré 4.

\section{Analogies entre l'expérience et la rétroaction de l'albédo de surface}

Les analogies entre 1'expérience proposée et le système climatique simple illustrant la «rétroaction de l'albédo» précédemment décrite sont les suivantes :

- l'analogue du moment de rappel créé par le contrepoids $m_{3}$ dans l'expérience est, pour le système climatique simplifié, l'émission de rayonnement qui augmente avec la température (loi de Planck). Lorsque les températures de la surface de la Terre et de l'atmosphère augmentent, toutes choses étant égales par ailleurs, la surface et l'atmosphère émettent plus de rayonnement dont une fraction atteint l'espace, ce qui refroidit le système Terre ;

- l'augmentation du moment de force due au transfert d'eau du réservoir haut vers le réservoir bas est analogue, pour le climat, à l'augmentation du flux solaire absorbé à cause de la diminution de l'albédo lorsque la température augmente. Si, pour une variation d'angle donnée, l'augmentation du moment de rappel créé par le contrepoids $m_{3}$ est supérieure à l'augmentation du moment de force due au transfert d'eau, alors le système est stable. Pour le climat, si l'augmentation, avec la température, du flux infrarouge émis par la surface de la Terre et l'atmosphère vers l'espace (le système Terre perd de l'énergie) est supérieure à l'augmentation du flux solaire absorbé (le système Terre gagne de l'énergie), alors le système climatique est stable. Dans le cas contraire, il devient instable, 
comme c'est aussi le cas si la masse du contrepoids $m_{3}$ est trop faible. Des analogies peuvent également être établies lorsque la rétroaction «sature»:

- l'analogue pour le climat du réservoir supérieur vide ne pouvant plus contribuer à la rétroaction positive est le cas où la neige et les glaces disparaissent complètement. Dans ce cas, il ne peut plus y avoir de fonte ;

- l'analogue du réservoir du bas qui est plein est le cas où la Terre serait entièrement recouverte par la glace. Dans ce cas, il ne peut plus y avoir d'extension des zones englacées.

L'exemple de la rétroaction de l'albédo permet de présenter de façon très simple le principe d'une rétroaction positive et de faire un parallèle direct avec l'expérience de la balance proposée ici. Le système climatique est infiniment plus compliqué, mais les concepts de base et les analogies que nous avons mentionnées restent néanmoins des guides de compréhension tout à fait valables.

\section{Le système climatique et ses rétroactions}

Comme d'autres planètes, la Terre est entourée d'une atmosphère. Celle de notre planète est constituée de $78 \%$ d'azote, $21 \%$ d'oxygène, $1 \%$ d'argon et de $0,04 \%$ de dioxyde de carbone ainsi que de quelques autres gaz à l'état de traces.

Dans l'explication de la rétroaction de l'albédo, nous avons abordé de façon simplifiée les échanges radiatifs du système Terre. Nous y revenons plus en détail pour présenter d'autres rétroactions climatiques liées à l'effet de serre. Le climat de la Terre est principalement déterminé par les échanges radiatifs entre l'énergie reçue du Soleil, sous forme de rayonnement de longueur d'onde essentiellement comprise entre 0,2 et 4 micromètres qui peut être réfléchie ou absorbée par l'atmosphère ou par la surface de la Terre, et le rayonnement infrarouge émis par le système Terre de longueur d'onde comprise entre 4 et 40 micromètres.

L'émission de rayonnement est régie par la loi d'émission du corps noir (loi de Planck): suivant cette loi, tout corps émet un rayonnement thermique dont la

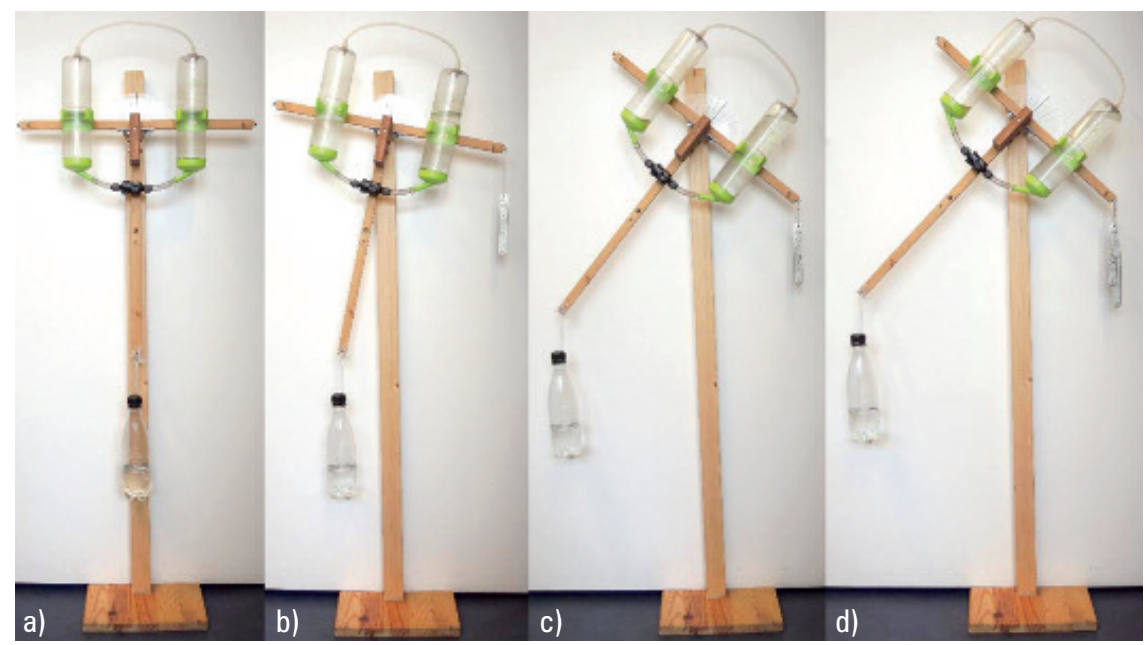

Figure 6. Dispositif expérimental avec un réglage de la force de rappel (légère diminution de la quantité d'eau dans la bouteille) conduisant à une saturation de la rétroaction: a) état initial, système non perturbé ; b) avec perturbation (masse $m_{1}=M$ ), le robinet est ouvert, les deux cylindres communiquent, nouvel état stable ; c) idem avec $m_{1}=2 M$, nouvel état stable, la rétroaction est saturée, le cylindre du haut est vide, l'eau ne peut plus couler ; d) idem avec masse $m_{1}=3 \mathrm{M}$ ). Puisque la rétroaction est saturée, l'angle est seulement légèrement modifié par rapport à (c) et l'effet de cette perturbation supplémentaire est équivalent à celui du cas où il n'y a pas rétroaction.

puissance et la fréquence augmentent avec la température. Lorsqu'il émet un rayonnement, tout corps perd de l'énergie. Lorsque le système Terre est à l'équilibre radiatif, l'énergie gagnée par absorption du rayonnement solaire est contrebalancée par l'énergie perdue par émission de rayonnement infrarouge.

Toute chose étant égale par ailleurs, une augmentation de température conduit à un accroissement du rayonnement infrarouge émis par la Terre, et donc à un accroissement de l'énergie perdue par le système. C'est le mécanisme essentiel pour stabiliser la température de surface d'une planète. Il est connu depuis longtemps et ses effets sont calculés avec précision: tous les modèles numériques de climat, qui sont des représentations numériques du fonctionnement du climat de la Terre, donnent dans ce cas des résultats quasiment identiques (figure $7 b$ ). Comme expliqué dans l'expérience décrite ci-dessus, c'est le rôle joué par le contrepoids, la petite bouteille en bas du bras vertical.

L'effet de serre est décrit dans un article de La Météorologie intitulé «L'effet de serre atmosphérique: plus subtil qu'on ne le croit!» (Dufresne et Treiner, 2011). L'équilibre radiatif du système Terre-atmosphère peut être perturbé par une modification, par exemple du rayonnement solaire incident ou des concentrations de gaz à effet de serre contenus dans l'atmosphère, tels le dioxyde de carbone, le méthane, l'ozone ou la vapeur d'eau, car ces gaz interagissent avec le rayonnement infrarouge sortant. Les activités humaines qui utilisent des combustibles fossiles dans le secteur de production d'énergie, les transports ou le secteur résidentiel, ainsi que la déforestation et les pratiques agricoles conduisent à des émissions de dioxyde de carbone et de méthane. En conséquence, les concentrations de ces gaz dans l'atmosphère augmentent et cela conduit à un déséquilibre du bilan d'énergie. On appelle forçage radiatif l'effet d'une telle perturbation sur le bilan radiatif au sommet de l'atmosphère, en supposant que toutes les autres caractéristiques de l'atmosphère et de la surface restent fixées.

Il est possible de calculer le forçage radiatif dû, par exemple, à l'augmentation de la concentration d'un gaz particulier. Pour un doublement de la concentration de dioxyde de carbone, l'émission de rayonnement thermique du système Terre vers l'espace diminuerait d'environ $4 \mathrm{Wm}^{-2}$. Le système Terre émettant alors moins de rayonnement qu'il n'absorbe de rayonnement solaire, il se réchauffe jusqu'à ce qu'un nouvel équilibre radiatif soit atteint. Pour un doublement de la concentration de dioxyde de carbone, l'élévation de la température à la surface de la Terre serait de $1,1 \mathrm{~K}$ si la seule réponse du système était une variation de 
température et si cette variation était uniforme dans l'atmosphère et n'affectait que la loi d'émission du corps noir. C'est ce que l'on appelle la réponse de Planck, car dans cette situation seule la fonction de Planck dépend de la température.

En réalité, le système climatique est un ensemble très complexe au sein duquel l'atmosphère, l'océan, l'hydrosphère, les surfaces continentales, la cryosphère et la biosphère interagissent en permanence en échangeant de l'eau, de l'énergie, du dioxyde de carbone, du méthane. Le climat de la Terre est déterminé par de nombreuses variables. Les échanges entre les différentes composantes du système climatique sont tels qu'un changement d'une variable caractérisant un milieu agit sur une variable d'un autre milieu; ils définissent ainsi de multiples couplages entre les différentes composantes du système climatique. En réponse à un doublement de la concentration de dioxyde de carbone, les modèles numériques de climat simulent une augmentation de température comprise entre 2 et $4,5 \mathrm{~K}$, bien plus grande que la valeur de $1,1 \mathrm{~K}$ mentionnée précédemment. Cette valeur tient compte de rétroactions positives d'ampleur dans le système climatique, parmi lesquelles on peut citer (voir également Bony et Dufresne, 2007 et Boucher, 2014):

- la rétroaction de la vapeur d'eau. La vapeur d'eau est un gaz qui absorbe fortement le rayonnement infrarouge émis par la Terre. Elle est le principal contributeur à l'effet de serre naturel. Comme le contenu maximum en vapeur d'eau de l'air augmente avec la température selon la loi de ClausiusClapeyron (Spiga, 2016), il s'ensuit qu'une augmentation de température entraîne une augmentation du contenu de l'atmosphère en vapeur d'eau, ce qui conduit à son tour à une augmentation de l'effet de serre, une augmentation de température et ainsi de suite ;

- la rétroaction des nuages. La couverture nuageuse et les propriétés microphysiques et radiatives des nuages sont sensibles aux variations climatiques. Les nuages ont deux effets opposés sur le climat: d'une part, ils réfléchissent une partie du rayonnement solaire, ce qui tend à refroidir la Terre et, d'autre part, ils absorbent le rayonnement infrarouge, ce qui tend à augmenter l'effet de serre et donc à réchauffer la Terre. Les nuages bas ont un fort albédo et contribuent
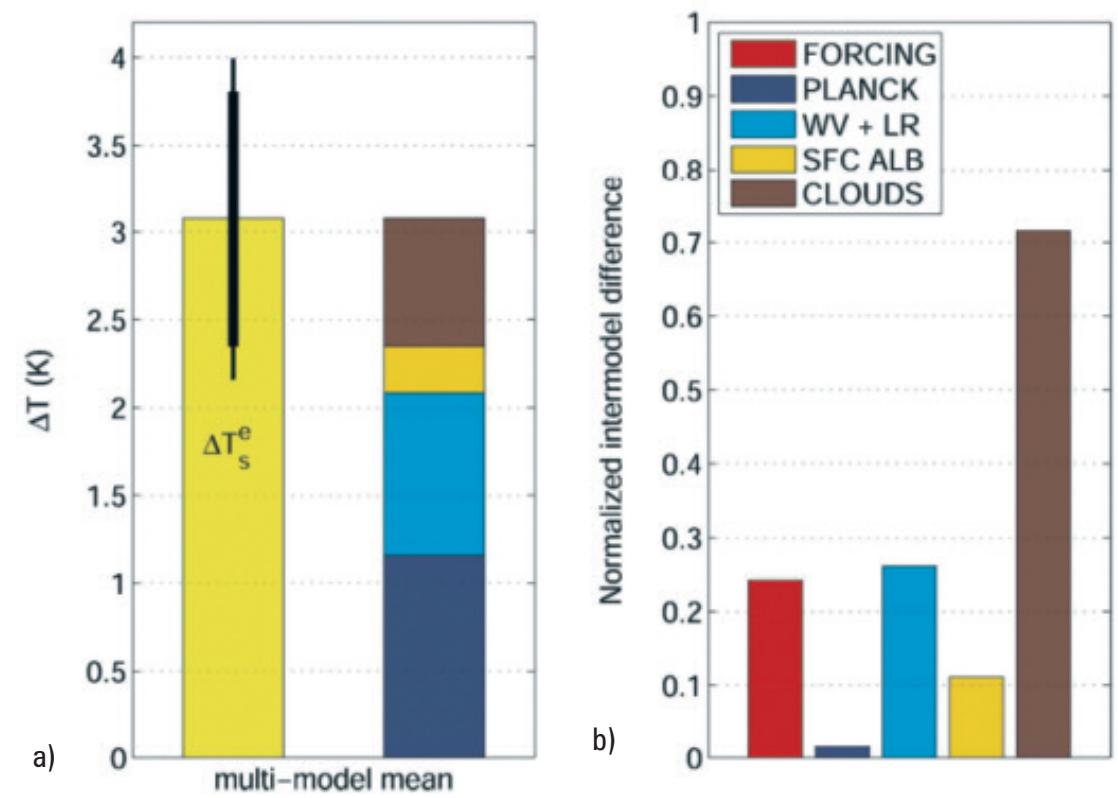

Figure 7. a) La barre verticale jaune représente le réchauffement moyen à l'équilibre simulé par 12 modèles climatiques pour un doublement de la concentration de dioxyde de carbone, la barre noire représentant la dispersion des valeurs entre ces modèles: \pm 1 écart type (gros trait) et intervalle de confiance 5-95\% (trait fin). La barre à droite représente les différentes contributions à ce réchauffement: la réponse de Planck (bleu foncé) et les différentes rétroactions dues à la vapeur d'eau et au gradient vertical de température (bleu clair), à l'albédo de surface (orange) et aux nuages (marron). b) Dispersion des valeurs entre ces modèles pour chacune de ces contributions, définie comme l'écart type entre les modèles pour chaque contribution divisé par l'écart type total. Les couleurs ont la même signification que précédemment, avec en plus une barre rouge pour représenter l'effet de l'incertitude sur la valeur du forçage pour un doublement de la concentration de $\mathrm{CO}_{2}$ (d'après Dufresne et Bony, 2008).

faiblement à l'effet de serre, ils ont donc un effet refroidissant. Pour les nuages hauts, l'effet de serre et l'albédo tendent à se compenser. On estime aujourd'hui que le réchauffement conduit à une diminution de la couverture de nuages bas (Boucher et al., 2013). Il s'ensuit donc que la rétroaction des nuages est en moyenne positive, même si sa valeur est encore très incertaine (voir figure $7 \mathrm{~b}$ ) ; cette rétroaction constitue la principale source d'incertitude de l'estimation de la sensibilité climatique, incertitude qui s'est peu réduite depuis les résultats des premiers modèles il y a une trentaine d'années ;

- la rétroaction de l'albédo de surface. Cette rétroaction, liée à la glace de mer et à la neige est l'une des raisons pour laquelle le réchauffement est plus marqué en Arctique qu'ailleurs.

En plus des rétroactions présentées ci-dessus, il existe de nombreuses autres rétroactions physiques, biogéochimiques, et notamment celles liées au cycle du carbone, aux changements de couvert végétal, à la libération du méthane contenu dans les océans ou sous le permafrost et à la chimie atmosphérique.

La figure 7 présente, pour un doublement de la concentration de dioxyde de carbone, le réchauffement simulé par douze modèles climatiques, les différentes contributions à ce réchauffement et la dispersion entre les modèles de ces estimations. On voit clairement l'importance des rétroactions puisque le réchauffement moyen global ( $3 \mathrm{~K}$ ) est environ trois fois plus élevé que la réponse de Planck $(1,1 \mathrm{~K})$, c'est-à-dire la réponse sans rétroaction. L'augmentation de la concentration en vapeur d'eau est la rétroaction qui contribue le plus au réchauffement, en moyenne, tandis que la rétroaction due aux nuages est celle qui contribue le plus à la dispersion des valeurs entre les modèles.

\section{Conclusion}

L'expérience proposée ici permet de visualiser le mécanisme d'une rétroaction positive et de comprendre les raisons et les conditions suivant lesquelles une rétroaction positive peut simplement amplifier l'effet d'une perturbation ou, au contraire, rendre le système instable, comme dans le cas où le contrepoids $m_{3}$ de l'expérience n'est pas assez lourd. Il est également 
possible de faire des analogies fortes entre les différents éléments de cette expérience et le système climatique. Par exemple, dans l'expérience, c'est la masse $m_{3}$ qui stabilise le système en créant un moment de rappel ; dans le système climatique, c'est la loi du corps noir (ou loi de Planck) en émettant davantage de rayonnement lorsque la température augmente.

Les rétroactions jouent un rôle clé dans la variabilité du climat et les changements climatiques, car elles amplifient fortement cette réponse dite «de Planck». La rétroaction - positive - due à la vapeur d'eau est celle qui amplifie le plus le réchauffement. Les processus physiques fondamentaux qui la gouvernent sont bien compris, cependant son importance justifie de poursuivre les recherches en matière d'observation et de modélisation pour affiner son estimation. Pour la rétroaction due aux nuages, la valeur de son amplitude, voire son signe, sont simulées très différemment suivant les modèles et il existe peu de guides théoriques ou expérimentaux pour contraindre cette valeur, même si des progrès ont récemment été réalisés. C'est la principale source d'incertitude pour les rétroactions physiques du climat et les projections climatiques en sont donc affectées.

La bonne estimation de l'amplitude $\mathrm{du}$ réchauffement est primordiale dans la mesure où l'essentiel des changements climatiques et de leurs conséquences en dépend. La compréhension des rétroactions, de leurs mécanismes, de leurs amplitudes et des échelles de temps sur lesquelles elles pourraient agir est cruciale pour mieux quantifier les risques des perturbations liées au réchauffement de la Terre et les horizons temporels auxquels les conséquences négatives se manifesteront.

\section{Représentation mathématique du dispositif expérimental et de l'expérience}

Soient (figure 4):

$m_{1}$, la masse accrochée au bras horizontal qui perturbe l'équilibre

$m_{2}$, la masse de chacun des deux cylindres contenant de l'eau accroché au bras horizontal

$\Delta m_{2}$, la masse supplémentaire d'eau dans le cylindre bas lorsque le robinet est ouvert

$m_{3}$, la masse de rappel accrochée en bas $d u$ bras vertical

$l_{1}$, la distance entre le point d'accrochage de la masse $m_{1}$ et le point 0

$I_{2}$, la distance entre les deux cylindres

$I_{3}$, la distance entre le point d'accrochage de la masse $m_{3}$ et le point 0

$\Delta \theta$, l'angle d'inclinaison du balancier par rapport à la verticale lorsque l'équilibre est perturbé

$\Delta \theta_{r}$, l'angle supplémentaire d'inclinaison du balancier par rapport à la verticale dû à la rétroaction lorsque le robinet est ouvert

$Z$, la différence de hauteur entre les niveaux d'eau des deux cylindres quand la rétroaction est activée

$M^{\mathrm{h}}$, le moment de la masse sur la barre horizontale, $M_{\mathrm{i}}^{\mathrm{h}}$, le moment initial, et $M_{r}^{\mathrm{h}}$ le moment supplémentaire dû à la rétroaction

$M^{\mathrm{r}}$, le moment de la masse de rappel

$\rho$, la masse volumique de l'eau

$S$, la section des cylindres.

Au départ, lorsque le système n'est pas perturbé, le balancier est tel que le bras long est vertical et l'angle $\Delta \theta$ est nul.

Lorsqu'une masse tourne autour d'un axe 0 , son moment angulaire est le produit de la force de gravité qui agit sur la masse $(m g)$ et de $I$, la distance par rapport à l'axe de rotation. Cette quantité est conservée et la somme des moments d'un système est nulle à l'équilibre.

À la suite de la perturbation provoquée par l'ajout de la masse $m_{1}$, le balancier est incliné d'un angle $\Delta \theta$, et les moments sont donnés par les formules suivantes:

- le moment de la masse de rappel s'écrit

$$
M^{r}=-g I_{3} m_{3} \sin (\Delta \theta)
$$

- et le moment initial de la masse sur la barre horizontale est

$$
M_{\mathrm{i}}^{\mathrm{h}}=g I_{1} m_{1} \cos (\Delta \theta)
$$

À l'équilibre, la somme des moments est nulle, $M^{r}+M_{i}^{h}=0$, ou encore:

$$
M_{\mathrm{i}}^{\mathrm{h}}-\mathrm{g} I_{3} m_{3} \sin (\Delta \theta)=0
$$

Pour $\Delta \theta$ suffisamment petit :

$$
\Delta \theta \approx \frac{l_{1} m_{1}}{l_{3} m_{3}}
$$

Quand on ouvre le robinet et que la rétroaction se met en place, l'inclinaison du balancier augmente de $\Delta \theta_{r}$ et le cylindre du bas reçoit une quantité d'eau supplémentaire $\Delta m_{2}$.

La différence de hauteur d'eau entre les deux cylindres est:

$$
Z=I_{2} \sin \left(\Delta \theta+\Delta \theta_{r}\right) \approx I_{2}\left(\Delta \theta+\Delta \theta_{r}\right)
$$

Le moment horizontal devient:

$M^{\mathrm{h}}=M^{\mathrm{h}}+M^{\mathrm{h}}$, où $M^{\mathrm{h}}$ rest le moment supplémentaire dû à la rétroaction qui s'écrit:

$$
M_{\mathrm{r}}^{\mathrm{h}}=\mathrm{g} I_{2} \Delta m_{2} \cos \left(\Delta \theta+\Delta \theta_{\mathrm{r}}\right)
$$

La masse d'eau qui est passée d'un cylindre à l'autre est la moitié de la différence entre les masses d'eau entre les deux cylindres:

$$
\Delta m_{2}=\frac{\rho S Z}{2}
$$

En combinant les équations (5) et (7), on obtient:

$$
\Delta m_{2} \approx \rho S I_{2} \frac{\Delta \theta+\Delta \theta_{\mathrm{r}}}{2}
$$

Le moment $M^{\mathrm{h}} \approx M_{\mathrm{i}}^{\mathrm{h}}+M_{\mathrm{r}}^{\mathrm{h}}$ se réécrit:

$$
M^{\mathrm{h}} \approx M_{\mathrm{i}}^{\mathrm{h}}+g \rho S I_{2}^{2} \frac{\Delta \theta+\Delta \theta_{\mathrm{r}}}{2}
$$

Compte tenu de la déviation supplémentaire due à la rétroaction, le moment de rappel de cet équilibre s'écrit:

$$
M^{r}=-g I_{3} m_{3} \sin \left(\Delta \theta+\Delta \theta_{r}\right)
$$

Le moment total étant nul à l'équilibre, et en combinant les équations (9) et (10), on obtient l'angle total de déviation du balancier lorsque la rétroaction est mise en action:

$$
\Delta \theta+\Delta \theta_{\mathrm{r}} \approx \Delta \theta\left(\frac{1}{1-\frac{\rho S I_{2}^{2}}{2 I_{3} m_{3}}}\right)
$$

\section{Bibliographie}

Bony S., Dufresne J.-L., 2007. Processus régissant la sensibilité climatique. La Météorologie, 56, 29-32.

Boucher 0., 2014. Utilisation d'un modèle de climat simplifié. La Météorologie, 87, 13-16.

Boucher O., Randall D., Artaxo P., Bretherton C., Feingold G., Forster P., Kerminen V.-M., Kondo Y., Liao H., Lohmann U., Rasch P., Satheesh S.K., Sherwood S., Stevens B., Zhang X.Y., 2013. Clouds and aerosols. In Climate change 2013: The physical science basis. Contribution of working group I to the fifth assessment report of the intergovernmental panel on climate change. Cambridge University Press

de Rosnay J., 1978. History of cybernetics and systems science. Disponible à l'adresse : http://pespmc1.vub.ac.be/CYBSHIST.html

Dufresne J.-L, Bony S., 2008. An assessment of the primary sources of spread of global warming estimates from coupled atmosphere-ocean models. J. Clim., 21, 5135-5144.

Dufresne J.-L., Saint-Lu M., 2016. Positive feedback in climate: stabilization or runaway illustrated by a simple experiment. Bull. Amer. Meteorol. Soc., 97, 755-765.

Dufresne J.-L., Treiner J., 2011. L'effet de serre atmosphérique: plus subtil qu'on ne le croit! La Météorologie, 72, 31-41.

Jacob F., 1970. La logique du vivant: une histoire de I'hérédité. Paris, Gallimard, 357 p.

Spiga A., 2016. Comment expliquer simplement la formation des nuages? La Météorologie, 95, 21-24 